Improving Order Picking Efficiency by Analyzing Combinations of Storage, Batching, Zoning, and Routing Policies

Peer-reviewed author version

VAN GILS, Teun; BRAEKERS, Kris; RAMAEKERS, Katrien; DEPAIRE, Benoit \& CARIS, An (2016) Improving Order Picking Efficiency by Analyzing Combinations of Storage, Batching, Zoning, and Routing Policies. In: Paias, Ana; Ruthmair, Mario; Voß, Stefan (Ed.). Computational Logistics; 7th International Conference, ICCL 2016, Lisbon, Portugal, September 7-9, 2016, Proceedings, Springer International Publishing,p. 427-442.

DOI: $10.1007 / 978-3-319-44896-1 \_28$

Handle: http://hdl.handle.net/1942/22615 


\title{
Improving Order Picking Efficiency by Analyzing Combinations of Storage, Batching, Zoning, and Routing Policies
}

\author{
Teun van Gils ${ }^{1} \bowtie$, Kris Braekers $^{1,2}$, Katrien Ramaekers ${ }^{1}$, Benoît Depaire ${ }^{1}$, and \\ An Caris ${ }^{1}$ \\ 1 Hasselt University, Agoralaan Building D, BE-3590 Diepenbeek, Belgium \\ 2 Research Foundation Flanders (FWO), Egmontstraat 5, 1000 Brussels, Belgium \\ \{teun.vangils, kris.braekers, katrien.ramaekers, benoit.depaire, \\ an.caris\}@uhasselt.be
}

\begin{abstract}
In order to differentiate from competitors in terms of customer service, warehouses accept late orders while providing delivery in a quick and timely way. This trend leads to a reduced time to pick an order. The objective of this research is to simulate and evaluate the interaction between several storage, batching, zone picking and routing policies in order to reduce the order picker travel distance. The value of integrating these four operation policy decisions is proven by a real-life case study. A full factorial ANOVA provides insight into the interactions between storage, batching, zoning, and routing policies. The results of the study clearly indicate that warehouses can achieve significant benefits by considering storage, batching, zone picking, and routing policies simultaneously. Awareness of the influence of an individual policy decision on the overall warehouse performance is required to manage warehouse operations, resulting in enhanced customer service.
\end{abstract}

Keywords: order picking; storage; order batching; zone picking; routing; warehouse policies interactions

\section{Introduction}

As customer markets globalize, supply chains are increasingly depending on efficient and effective logistical systems to distribute products across a large geographical area. Warehouses are important parts of supply chains, and therefore warehouse operations need to work in an efficient and effective way. A warehouse can be defined as a facility where activities of receiving, storage, order picking, and shipping are performed [9].

Order picking management, in particular organizing efficient and flexible order picking systems, has been identified as an important and complex planning operation. In order to differentiate from competitors in terms of customer service, warehouses accept late orders from customers while providing delivery in a quick and timely way. By accepting late orders, the remaining time to pick 
an order is reduced. Furthermore, the order behavior of customers has changed from ordering few and large orders to many orders consisting of only a limited number of order lines [6]. The changed order behavior can be ascribed to upcoming e-commerce markets and forces warehouses to handle a larger number of orders, while order picking time has shortened.

Four operational policy decisions can be distinguished with respect to order picking: storage location assignment, order batching, zone picking, and routing. In this paper several policies for each decision are considered and potential interactions between these decisions are investigated in order to manage order picking operations more efficiently. While the number of publications dealing with one specific order picking policy decision is extensive [6,9], only a limited number of researchers examine different decisions simultaneously (e.g. $[12,13,15,17]$ ), even though the efficiency of different order picking policy decisions seems to be interdependent [9]. The effect of zoning in combination with other order picking decisions, such as storage, routing and batching, has received especially little research attention. Therefore, the study's main objective is to analyze storage, batching, zoning, and routing in order to minimize the distance traveled by order pickers, with particular emphasis on the relation between these four order picking decisions. To the best of our knowledge, this study is the first to analyze the interaction of the four main operational order picking policy decisions (i.e. storage, batching, zoning, and routing).

The main contribution of this paper is the integration of storage, order batching, zone picking, and routing in order to improve order picking activities of a real-life warehouse. Furthermore, insights into the interactions between the four operational policy decisions are provided by performing a full factorial analysis of variance (ANOVA). It determines the impact of storage location assignment, order batching, zone picking, and routing on the distance traveled by order pickers, as well as the relation between each of the four order picking policy decisions.

The remainder of the paper is organized as follows. Section 2 is devoted to describe the context of the problem. In Sect. 3, the case study and the assumptions linked to the case are described. Subsequently, the experimental design is presented in Sect. 4, followed by the empirical results of the real-life case in Sect. 5. Managerial implications of this study are discussed in Sect. 6. Finally, Sect. 7 is devoted to the concluding remarks and future research directions.

\section{Problem Context}

Order picking as a warehouse function arises because goods are received in large volumes and customers order small volumes of different products. Each customer order is composed of one or more order lines, with every order line representing a single stock keeping unit (SKU) [6]. In order to manage order picking operations, warehouse managers are confronted with four operational decisions, in particular storage location assignment, order batching, zone picking, and routing.

The storage location assignment problem can be defined as determining the physical location at which incoming products are stored. One way to obtain a 
more efficient order picking process is to allocate fast moving products to storage locations closely located to the depot, rather than randomly assigning SKUs to storage locations. As traveling in a warehouse is often the dominant factor in order picker's activities, the travel distance reduction resulting from turnover based storage location assignment policies, will contribute to a more efficient order picking process. A turnover based storage assignment policy defines product classes by some measure of demand frequency of the product. Within-aisle storage, where all products in a pick aisle belong to the same class, across-aisle storage, where each product class is located across several pick aisles, diagonal storage, where product classes are located with respect to the depot, and perimeter storage where product classes are located around the perimeter of the warehouse are frequently used policies to locate the product classes in the order picking area [17].

Furthermore, batch picking, instead of picking each order separately, allows warehouses to handle a larger number of orders in shorter time windows. By picking multiple orders in a single picking tour, the order picker travel distance per order will be reduced. The order batching problem is concerned with deciding on rules defining which orders to combine on a pick list in order to minimize the order picker travel distance. The most straightforward algorithm for creating pick lists is a priority rule based algorithm, in which orders are prioritized and assigned to pick lists based on their priority (e.g. first-come-first-served (FCFS)). Seed algorithms generate batches by selecting an initial seed order (e.g. select the smallest order), after which unassigned customer orders are added to the seed order according to an order congruency rule (e.g. add an order such that the number of additional pick locations is minimal). Three other order batching heuristics can be distinguished: savings algorithms, data mining approaches, and metaheuristics. The reader is referred to [11] for an extensive overview of order batching algorithms.

Another practice of moving to a more efficient order picking process is dividing a warehouse into different smaller areas, being order picking zones. In contrast to strict order picking, in which order pickers are allowed to retrieve SKUs in the entire order picking area, each order picker is assigned to a single zone and responsible for picking all SKUs of an order belonging to this zone. As a consequence each order picker travels in a pre-specified part of the warehouse and thus travel time will be reduced. The assignment of items to different zones is mainly based on physical properties of products such as size and weight. Other allocation policies that may be considered are based on product demand properties, such as customer type and order frequency. If order integrity is violated (i.e. customer orders are split into separate pick lists), additional sorting activities are required to consolidate orders after retrieving the SKUs [14].

Finally, the purpose of considering routing policies is to sequence the items on the pick list in order to reduce the order picker travel distance. The problem of routing order pickers in a warehouse is mainly solved by using heuristics. The routing problem cannot be solved to optimality for every warehouse layout within reasonable computation times. A growing number of picking aisles, 
or larger pick lists result in fast increasing computing times. Furthermore, optimal routes may seem illogical to order pickers, resulting in deviations from the specified optimal routes. As an alternative to the optimal route, several more straightforward routing heuristics are proposed in the literature, including aisle-by-aisle, traversal or S-shape, return, midpoint, and largest gap. The aisleby-aisle routing policy is the most straightforward routing method, where order pickers visit every pick aisle containing at least one pick location through the entire length. Traversal routes are closely related to aisle-by-aisle routes. Order pickers only traverse every subaisle (i.e. the part of a pick aisle that is within one warehouse block) containing at least one pick location through the entire length. Another straightforward routing policy is the return strategy, where order pickers enter and leave each aisle containing at least one pick location from the same end. A midpoint routing policy extends the return strategy by requiring the order picker to return if he reaches the aisle midpoint. At the midpoint, the order picker returns and leaves the aisle from the same end as he entered the aisle. The largest gap strategy is similar to the midpoint strategy except that a picker enters an aisle only as far as the start of the largest gap within an aisle, instead of the midpoint. The largest gap is defined as the maximum distance between any two adjacent pick locations within a single aisle, or the maximum distance between an aisle end and a pick location $[16,18]$.

Previous research has focused on either storage, batching, zoning, or routing. The number of studies analyzing interactions between these operational warehouse policies are limited. Several simulation studies analyze combinations of two operational order picking policies (e.g. storage-routing [16, 17], zoning-batching [21], and routing-batching $[20,3,4])$, while $[2,13,12,15]$ investigate the combination of storage, batching, and routing policies. This paper differs from previous studies by analyzing the four main operational policy decisions simultaneously.

\section{Case Study}

In order to analyze the effect of integrating storage, batching, zoning, and routing, real-life data are used. The case study is based on a large warehouse located in Belgium. The warehouse stores approximately 90.000 SKUs on a surface of 30.000 square meter. In accordance with the large majority of order picking systems in Western Europe, the warehouse is fully manually operated. Automated picking systems are only useful in case of valuable, small and delicate products [6]. These kind of products are limited in the warehouse under consideration.

Strict order picking is currently applied in combination with random storage location assignment. Customer orders are transformed into pick lists according to the FCFS rule. Order pickers follow the aisle-by-aisle routing policy to retrieve all items on the pick list. The policy combination of random storage, FCFS batching, strict order picking, and aisle-by-aisle routing is used as benchmark in order to evaluate other storage, batching, zoning, and routing policies. As discussed before, choosing the optimal combination of different order picking 
policies is crucial for warehouse managers in order to minimize the distance traveled by order pickers and consequently reduce the order throughput time.

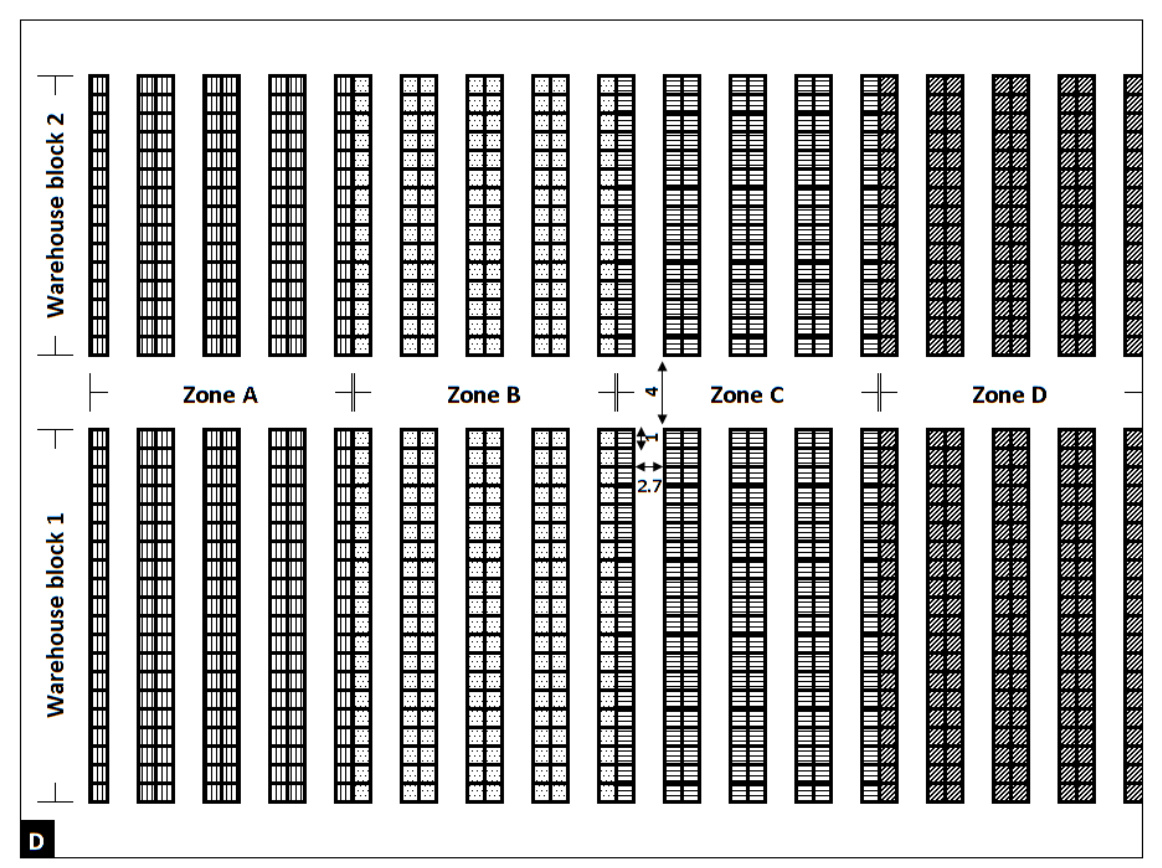

Fig. 1. Warehouse layout

The warehouse under consideration is shown in Fig. 1. The traditional multipleblock warehouse layout is frequently used in practice [17], making results of the study easily transferable to other warehouses. Furthermore, cross aisles have proven to result in significant efficiency benefits [18]. Besides traditional warehouse layouts with parallel pick aisles and straight middle aisles, alternative warehouse layouts, such as fishbone designs, can improve the order picking performance in case of a small number of items on the pick list [1]. As the batch capacity is limited to 26 orders in our problem setting, the fishbone design will likely be outperformed by the traditional warehouse layout. The warehouse in the simulation experiment has the following properties:

- The order picking area is divided into two warehouse blocks, each consisting of 16 picking aisles. The picking aisles are two-sided and wide enough for twoway travel. However, crossing the aisle is required in order to pick items from both sides of the same aisle, as the aisle width is 2.7 meter. The dimensions of the aisles, as well as the warehouse block configuration and the zone configuration (in case zone picking is applied) are shown on Fig. 1. 
- Order picking is completed manually using a picking vehicle with a capacity of 26 orders.

- Each picking tour starts and ends at the decentralized depot. The depot is marked as D in the bottom left corner of Fig. 1.

- A sort-while-pick strategy is used, maintaining order integrity, so that no downstream sorting is required. The picking vehicle is able to sort 26 different orders during a pick tour.

- All storage locations have an equal size.

In order to evaluate the order picking policies, the average travel distance is used as performance criterion. The average travel speed in both cross aisles and pick aisles is assumed to be equal. So, minimizing the distance traveled by order pickers is equivalent to minimizing the average travel time of order pickers, reducing the required labor hours for picking a particular number of orders. Order pickers are assumed to be able to traverse the aisles in both directions and to be able to change direction within the aisles.

\section{Experimental Design}

The objective of this research is to reduce the order picker travel distance, which results in a more efficient order picking process, by simulating and evaluating combinations of storage, batching, zone picking, and routing policies. Simultaneously analyzing storage location assignment, order batching, zone picking, and routing policies using a factorial design provides insights into the impact of each operational order picking decision on the distance traveled by order pickers, as well as into the relation between the operational order picking decisions. In the experiments of this paper five different storage location assignment policies, two order batching policies, five zone picking policies, as well as five routing policies are analyzed. The four factors and their associated factor levels are summarized in Table 1. The baseline scenario of this experiment is indicated in italic.

Table 1. Experimental factor setting

\begin{tabular}{ll}
\hline Factor & Factor levels (number of levels) \\
\hline Storage (S) & Random; across-aisle; within aisle; diagonal; perimeter (5) \\
Batching (B) & FCFS; seed (2) \\
Zone picking (Z) & Strict; 2 zones (CT); 2 zones (PF); 4 zones (CT); 4 zones (PF) (5) \\
Routing (R) & Aisle-by-aisle; traversal; return; largest gap; optimal (5) \\
\hline
\end{tabular}

$\mathrm{CT}=$ storage zone assignment based on customer type

$\mathrm{PF}=$ storage zone assignement based on pick frequency

Besides randomly assigning SKUs to storage locations, four turnover based storage location assignment policies are simulated, in particular across-aisle assignment, within-aisle assignment, diagonal assignment and assigning SKUs 
across the perimeter of the order picking area. Within each product class, in case of turnover based storage location assignment, each SKU is randomly assigned to only one storage location.

The currently used FCFS batching policy actually results in a random creation of pick lists in terms of travel distance, as FCFS batching does not take the location of SKUs in the order picking area into account. A seed order batching algorithm is used as an alternative to create batches. The order that requires the smallest number of picking aisles to visit, is selected as seed order. Next, the order that minimizes the number of additional aisles to visit in the route is added to the pick list. This algorithm is repeated until the batch contains 26 orders. Subsequently a new seed order is selected. The combination of this seed order selection rule and this accompanying order selection rule have yielded good results for different storage location assignment and routing policies [13,12].

Strict order picking is compared to four zone picking policies. Both the number of zones as well as the storage zone assignment policy should be determined in case of zone picking. In the simulation experiments, the warehouse is divided into either two or four order picking zones, and SKUs are assigned to order picking zones based on customer type (CT) or pick frequency (PF). This results in four additional zone picking policies.

In addition to the aisle-by-aisle routing heuristic, the travel distance for return, traversal, largest gap, and the optimal route is computed. As the routing problem cannot be solved to optimality for a multiple-block warehouse in reasonable computing times, the Lin-Kernighan-Helsgaun (LKH) heuristic for the traveling salesman problem (TSP) is used to approximate the optimal route [10]. The LKH heuristic has shown to provide excellent results, both in a general TSP context, and in the context of routing order pickers in a warehouse. Theys et al. [19] reported an average optimality gap of $0.1 \%$ for different warehouse settings.

To sum up, the simulation experiment consists of 250 possible combinations of policies (i.e. five storage location assignment policies $\times$ two batching policies $\times$ five zone picking policies $\times$ five routing policies). To reduce the stochastic effect from order generation, 30 replications per policy combination are performed, resulting in 7,500 observations. During each replication, all combinations of storage, batching, zoning and routing are tested on the same 1,690 randomly generated orders. Order sizes follow an exponential distribution with mean of 2.65 order lines. This factorial setting results in a $5 \times 2 \times 5 \times 5$ full factorial design.

The results of the simulation experiments are analyzed by a full factorial ANOVA. The assumptions under which the ANOVA F statistic is reliable, are independent observations, homogeneity of variance, as well as normally distributed observations. For each replication, all combinations of storage, batching, routing and zoning are simulated on the same randomly generated orders in order to stress the effects of policy decisions. Consequently, the 7,500 observations are not independently and a repeated measures ANOVA with storage, batching, routing, and zoning as within-subjects factors is required to analyze the main and interaction effects of the policy decisions [5]. Since the homogeneity assumption is violated, the F-test Type I error rate increases. The Greenhouse-Geisser (G- 
G) correction of the degrees of freedom is used to compensate for the increased F-test type I error rate. The G-G adjustment is the most conservative correction to compensate for the violation of sphericity $[5,8]$. In order to ensure the last ANOVA assumption (i.e. normality), the experimental design is balanced. The $\mathrm{F}$ statistic is quite robust to violations of normality when group sizes are equal [5]. In the simulation experiments in this paper, a balanced $5 \times 2 \times 5 \times$ 5 full factorial repeated measures ANOVA, with storage, batching, zoning, and routing as the within-subjects factors, is used to prove the value of studying the four operation policy decisions in an integrated manner.

\section{Empirical Results}

First, results of the repeated measures ANOVA are discussed in Sect. 5.1. Subsequently, the impact of each individual operational order picking policy decision, as well as the interaction effects between policy decisions are analyzed in Sect. 5.2 and 5.3 respectively.

\subsection{ANOVA Results}

In order to get a first insight into the results of the simulation experiments, the route lengths of the different factor combinations are analyzed by a $5 \times 2 \times$ $5 \times 5$ full factorial repeated measures ANOVA on average travel distance. The results of the repeated measures ANOVA are shown in Table 2. The first three columns show the sum of squares, the G-G degrees of freedom and the resulting mean square for the main and interactions effects, as well as for the residuals. The last two columns are devoted to the $\mathrm{F}$ statistic and the p-value for testing the statistical significance of storage, batching, zoning, and routing, as well as all interactions between the four operational policy decisions.

Table 2 indicates that the main effects of storage location assignment, order batching, zoning and routing are statistically significant. This means that there is a significant difference between the five storage location policies, the two order batching policies, the five zoning policies, as well as the five different routing policies on the average distance traveled by order pickers, respectively. The decision on which storage, which batching, which zoning, and which routing policy to use does influence the average route length.

Furthermore, Table 2 shows that all factors in the simulation experiment are significantly interacting with each other. All of the six two-way interactions, all three-way interactions, as well as the four-way interaction between storage, batching, zoning and routing are statistically significantly different form zero. As three out of the four factors in the experiment contain five levels, the 30 replications give rise to a large number of observations. Null hypotheses are much easier rejected with a large number of factor levels and a large number of observations because of a greater probability that one of the factor levels is interacting with another factor level [7]. However, the ANOVA shows strong statistically effects, both for the main effects and the interaction effects. Given 
Table 2. $5 \times 2 \times 5 \times 5$ full factorial repeated measures ANOVA on average travel distance

\begin{tabular}{|c|c|c|c|c|c|}
\hline & Sum of squares & $\mathrm{df}$ & Mean square & $\mathrm{F}$ & p-value \\
\hline \multicolumn{6}{|l|}{ Main effects } \\
\hline Storage (S) & $26,895,933,301$ & 2.91 & $9,237,614,573$ & $13,853.95$ & 0.000 \\
\hline Batching (B) & $181,937,289,935$ & 1.00 & $181,937,289,935$ & $284,683.12$ & 0.000 \\
\hline Zoning $(\mathrm{Z})$ & $358,908,254,822$ & 2.57 & $139,604,086,655$ & $60,486.02$ & 0.000 \\
\hline Routing (R) & $79,222,871,786$ & 2.18 & $36,318,042,321$ & $229,618.82$ & 0.000 \\
\hline \multicolumn{6}{|l|}{ Two way interaction } \\
\hline $\mathrm{S} \times \mathrm{B}$ & $11,070,135$ & 3.27 & $3,383,774$ & 11.14 & 0.000 \\
\hline $\mathrm{S} \times \mathrm{Z}$ & $16,831,331,146$ & 7.61 & $2,211,840,545$ & $3,526.56$ & 0.000 \\
\hline $\mathrm{S} \times \mathrm{R}$ & $8,595,751,592$ & 7.03 & $1,222,476,423$ & $11,468.01$ & 0.000 \\
\hline $\mathrm{B} \times \mathrm{Z}$ & $16,416,356,030$ & 2.91 & $5,634,479,183$ & $9,634.53$ & 0.000 \\
\hline$B \times R$ & $5,391,003,315$ & 2.74 & $1,966,180,751$ & $47,170.76$ & 0.000 \\
\hline $\mathrm{Z} \times \mathrm{R}$ & $12,905,574,845$ & 6.00 & $2,152,147,805$ & $18,137.89$ & 0.000 \\
\hline \multicolumn{6}{|l|}{ Three way interaction } \\
\hline $\mathrm{S} \times \mathrm{B} \times \mathrm{Z}$ & $621,242,209$ & 8.43 & $73,716,968$ & 179.82 & 0.000 \\
\hline $\mathrm{S} \times \mathrm{B} \times \mathrm{R}$ & $505,815,157$ & 8.47 & $59,717,570$ & $1,132.60$ & 0.000 \\
\hline $\mathrm{S} \times \mathrm{Z} \times \mathrm{R}$ & $6,383,449,026$ & 13.79 & $463,008,935$ & $2,894.19$ & 0.000 \\
\hline $\mathrm{B} \times \mathrm{Z} \times \mathrm{R}$ & $703,844,710$ & 6.98 & $100,770,162$ & $1,642.05$ & 0.000 \\
\hline \multicolumn{6}{|l|}{ Four way interaction } \\
\hline $\mathrm{S} \times \mathrm{B} \times \mathrm{Z} \times \mathrm{R}$ & $478,689,655$ & 15.11 & $31,682,633$ & 330.12 & 0.000 \\
\hline \multicolumn{6}{|l|}{ Residuals } \\
\hline Between subjects & $330,171,235$ & 29.00 & $11,385,215$ & & \\
\hline Within S & $56,300,343$ & 84.44 & 666,786 & & \\
\hline Within B & $18,533,524$ & 29.00 & 639,087 & & \\
\hline Within Z & $172,078,430$ & 74.56 & $2,308,039$ & & \\
\hline Within R & $10,005,553$ & 63.26 & 158,167 & & \\
\hline Within $\mathrm{S} \times \mathrm{B}$ & $28,813,836$ & 94.87 & 303,705 & & \\
\hline Within $\mathrm{S} \times \mathrm{Z}$ & $138,409,444$ & 220.68 & 627,196 & & \\
\hline Within $\mathrm{S} \times \mathrm{R}$ & $21,736,707$ & 203.91 & 106,599 & & \\
\hline Within $\mathrm{B} \times \mathrm{Z}$ & $49,413,333$ & 84.49 & 584,821 & & \\
\hline Within $\mathrm{B} \times \mathrm{R}$ & $3,314,322$ & 79.51 & 41,682 & & \\
\hline Within $\mathrm{Z} \times \mathrm{R}$ & $20,634,248$ & 173.90 & 118,655 & & \\
\hline Within $\mathrm{S} \times \mathrm{B} \times \mathrm{Z}$ & $100,189,592$ & 244.39 & 409,950 & & \\
\hline Within $\mathrm{S} \times \mathrm{B} \times \mathrm{R}$ & $12,951,295$ & 245.63 & 52,726 & & \\
\hline Within $\mathrm{S} \times \mathrm{Z} \times \mathrm{R}$ & $63,962,727$ & 399.82 & 159.979 & & \\
\hline Within $\mathrm{B} \times \mathrm{Z} \times \mathrm{R}$ & $12,430,509$ & 202.55 & 61,369 & & \\
\hline Within $\mathrm{S} \times \mathrm{B} \times \mathrm{Z} \times \mathrm{R}$ & $42,053,031$ & 438.16 & 95,977 & & \\
\hline Total & \multicolumn{5}{|c|}{$716,889,475,792 \quad 7,499.00$} \\
\hline
\end{tabular}

the significance of the effects, the main and interaction effects are examined in more detail in the next sections.

\subsection{Main Effect of Storage, Batching, Routing and Zone Picking}

Table 3 summarizes the average route length in meters for each operational policy decision over all combinations with other policies, as well as the relative difference between each order picking policy and the average best performing policy within each operational decision area. Additionally, the statistical significance of all levels of the different experimental factors are analyzed using a Bonferroni ttest. The Bonferroni method seems to be the most robust technique in terms of 
Table 3. Average travel distance for each operational order picking policy (in meter)

\begin{tabular}{|c|c|c|c|c|c|c|c|c|}
\hline & Storage & Mean & Batching & Mean & Zoning & Mean & Routing & Mean \\
\hline \multirow[t]{2}{*}[1]{} & Within-aisle & $25,831.1$ & Seed & $23,016.4$ & 4 zones $(\mathrm{CT})$ & $19,916.0$ & Optimal & $22,814.0$ \\
\hline & Diagonal & $26,220.9$ & FCFS & $32,866.9$ & 4 zones $(\mathrm{PF})$ & $24,577.2$ & Traversal & $26,945.9$ \\
\hline Gap & $\operatorname{over}[1](\%)$ & 1.5 & & 30.0 & & 19.0 & & 15.3 \\
\hline & Across-aisle & $27,283.4$ & Mean & $27,941.6$ & 2 zones $(\mathrm{CT})$ & $25,355.3$ & Largest gap & $27,035.7$ \\
\hline Gap & $\operatorname{over}[1](\%)$ & 5.3 & & & & 21.5 & & 15.6 \\
\hline & Random & $30,142.2$ & & & 2 zones $(\mathrm{PF})$ & $29,489.9$ & Return & $31,405.2$ \\
\hline Gap & over [1] (\%) & 14.3 & & & & 32.5 & & 27.4 \\
\hline & Perimeter & $30,230.6$ & & & Strict & $40,369.7$ & Aisle-by-aisle & $31,507.4$ \\
\hline Gap & $\operatorname{over}[1](\%)$ & 14.6 & & & & 50.7 & & 27.6 \\
\hline & Mean & $27,941.6$ & & & Mean & $27,941.6$ & Mean & $27,941.6$ \\
\hline
\end{tabular}

power and control of the Type I error rate for evaluating multiple hypotheses [7]. The test results are summarized in Table 4. If two order picking policies are listed in the same subset in table 4 , differences fail to be statistically significant. The simulation results of storage location assignment policies, order batching policies, routing policies and zoning policies are discussed independently below.

Table 4. Post hoc multiple Bonferroni t-test for each operational policy decision on average travel distance (familywise error rate $=0.01$ )

\begin{tabular}{|c|c|c|c|}
\hline Storage & Batching & Zoning & Routing \\
\hline Within-aisle & Seed & 4 zones $(\mathrm{CT})$ & Optimal \\
\hline Diagonal & FCFS & 4 zones $(\mathrm{PF})$ & Traversal \\
\hline Across-aisle & & 2 zones $(\mathrm{CT})$ & Largest gap \\
\hline Random & & 2 zones $(\mathrm{PF})$ & Return \\
\hline Perimeter & & Strict & Aisle-by-aisle \\
\hline
\end{tabular}

The within-aisle storage location assignment policy is on average the best performing method for assigning SKUs to individual storage locations, followed by the diagonal and across-aisle storage policy. Except for the perimeter storage location assignment policy, all turnover based storage policies (i.e. within-aisle, across-aisle, and diagonal) are able to outperform the random assignment of SKUs to storage locations. These three turnover based storage policies are in the three top subsets in Table 4 and result in statistically significantly shorter travel distances compared to the random and perimeter storage location assignment policy. Random and respectively perimeter assignments yield on average $14.3 \%$ and $14.6 \%$ larger route lengths compared to the best performing method. These two storage policies form a single subset in the Bonferroni t-test, indicating that the average travel distance is on average not statistically significantly different. 
Two subsets are composed by evaluating the two batching policies (Table 4), giving evidence that the mean route length is statistically significantly different for each order batching policy. Simulations show that the seed order batching policy on average results in $30 \%$ shorter route lengths compared to FCFS batching (Table 3). This result is rather obvious as the seed rules take the product locations of each order into account in composing pick lists, while FCFS batching results in a random composition of pick lists in terms of travel distances.

The policy of dividing the warehouse into order picking zones outperforms the strict order picking policy. The average travel distance halves when changing from strict order picking to the best performing zone picking policy, i.e. the customer type storage zone assignment in which the order picking area is divided into four order picking zones. All sixteen picking aisles can be visited in a single pick tour in case of strict order picking, while a maximum of either eight or four aisles should be entered if the warehouse is respectively divided into two or four order picking zones. So each order picker only traverses a small part of the order picking area in order to retrieve all items on the pick list.

Tables 3 and 4 show that the optimal routing policy results in the smallest average distance traveled by order pickers. The optimal routing procedure is in the top performing subset as items on the pick list are sequenced in order to minimize the route length. The optimality gap for the four routing heuristics (i.e. traversal, largest gap, return, and aisle-by-aisle) is on average $15.3 \%, 15.6 \%$, $27.4 \%$, and $27.6 \%$ respectively. The results of the simulation experiments show statistically significant differences between all routing policies. However, the average route length difference between traversal and largest gap, as well as the mean difference between return and aisle-by-aisle are rather limited. Return and aisle-by-aisle routes are the most straightforward and worst performing routing heuristics. The traversal routing policy outperforms the aisle-by-aisle heuristic, because the traversal routing policy allows order pickers to leave an aisle in the middle cross-aisle, which results in shorter routes. The largest gap heuristic extends the return routing policy by requiring the order picker to return as he reaches the largest gap within an aisle. Consequently, largest gap routes outperform routes in which the order picker always returns to the middle cross-aisle.

\subsection{Interaction Effects}

The results of the simulation experiments are graphically illustrated in Fig. 2, disaggregated into combinations of storage location assignment policies, order batching policies, zoning policies, and routing policies. The interaction plot shows all two-way interactions between the four operational order picking policies. The lines on the graph illustrate the average travel distance for a particular order picking policy combination. The three graphs in the first column show the average travel distance in function of the different storage location assignment policies per batching, zoning and routing policy in the respective first, second and third graph of the first column. The graphs in the three remaining columns illustrate the average route length in function of the different batching, zoning and routing policies in an equivalent way. Most lines on the graphs converge and 
some even cross. For example, the lines of the aisle-by-aisle routing heuristic and the return policy are crossing on the graph illustrating the interaction between batching and routing, while the return, traversal, and largest gap routing heuristic converge when changing from the FCFS to the seed batching policy. The converging and crossing lines are in accordance with the results of the ANOVA in Table 2, indicating that there are strong interactions between the different operational order picking decisions.

The significant interactions between the different order picking policies originate from the fact that some combinations of warehouse policies yield excellent performances (e.g. perimeter storage assignment in combination with largest gap routing), while other combinations result in large average travel distances (e.g. FCFS batching in combination with return routing). From Fig. 2, the combination of the perimeter storage policy and the largest gap routing policy is an example of a well performing combination. Since fast moving SKUs are stored along the periphery of the warehouse blocks and the largest gap routes tend to follow the periphery of the warehouse, this combination of order picking policies outperforms other combinations of routing heuristics and perimeter storage location assignment. Aisle-by-aisle, traversal, as well as return routes show a strong increase in travel distance in combination with the perimeter storage compared to other storage location assignment policies.

Combinations of the straightforward routing policies (i.e. aisle-by-aisle and return) with FCFS batching appear to be inefficient. FCFS batching, which in fact results in a random creation of batches, generates pick lists with SKUs located in a large number of aisles and SKUs are diffused within each aisle. Aisleby-aisle routes can work efficiently only if the number of aisles to be visited is minimized, while return routes aim to minimize the travel distance within a pick aisle. This results in a large travel distance when combining FCFS batching with either the aisle-by-aisle or return routing policy. The average route length difference between FCFS and seed batching is much larger when combined with aisle-by-aisle and return routing compared to other routing methods.

In addition to some excellent performing and some inefficient combinations, the statistically significant interaction can be further explained by the fact that shifting from a bad performing factor level to a good performing level within the same factor results in much smaller performance benefits when other order picking policies are already efficiently performed compared to the situation in which other order picking policies on average result in large travel distances. For example the effect of different storage location assignment policies is not consistent over all levels of zoning. By dividing the warehouse into order picking zones, the effect of shifting to a more efficient storage policy on the route length is reduced compared to the strict order picking policy. The reason for this significant interaction term can be found in the smaller area that is crossed by order pickers to retrieve all items on the pick list in case of two or four order picking zones, as well as in case of turnover based storage location assignment. Zoning policies as well as storage policies aim to increase the density of SKUs retrieved in each aisle. Consequently, the performance impact resulting from changing the 


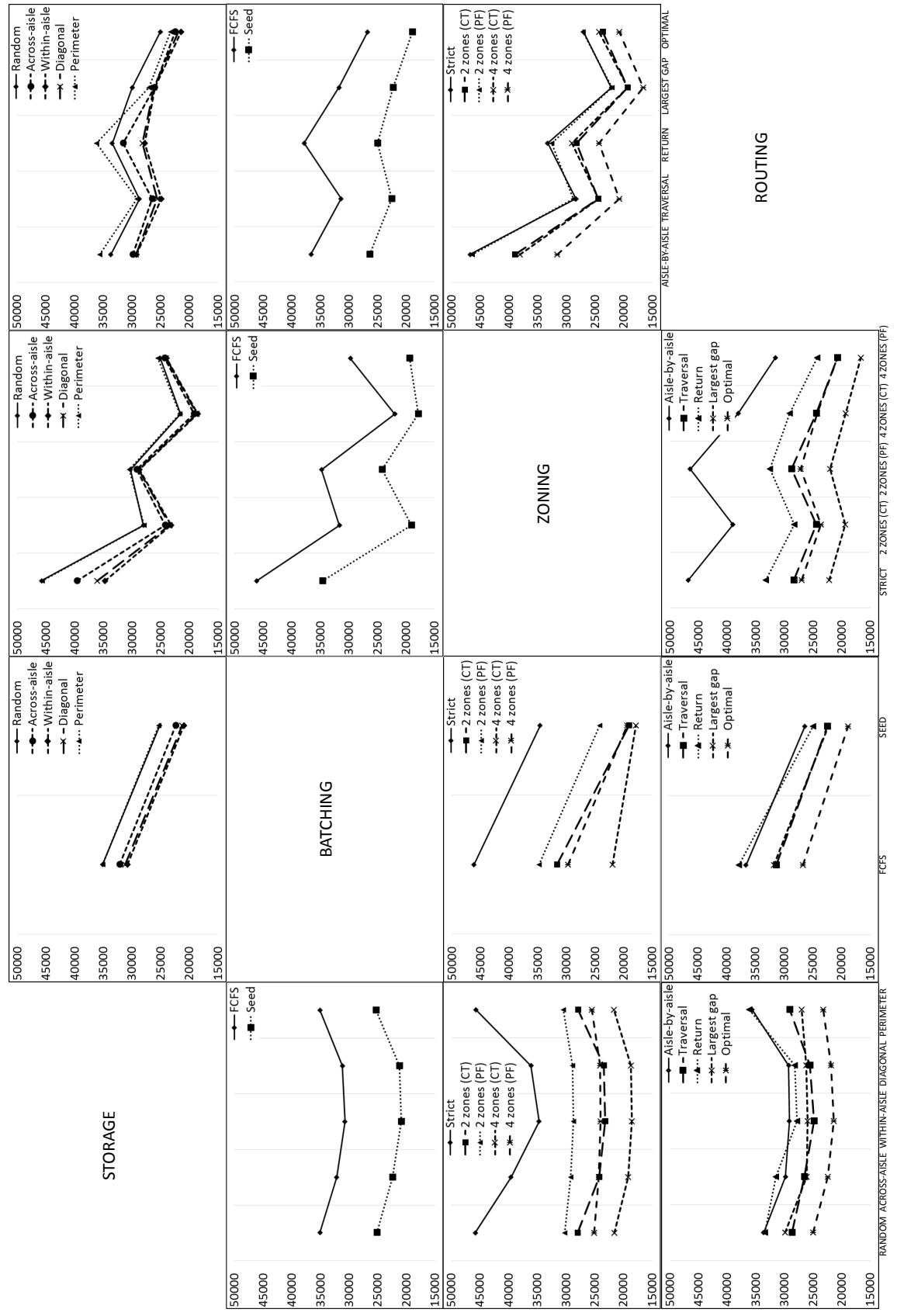

Fig. 2. Average travel distance in meter for each combination of storage, batching, zoning and routing policy 
storage policy is far greater in combination with strict order picking, compared to other zone picking policies.

\section{Managerial Implications}

The results of the simulation experiments show the importance of storage, batching, zoning, and routing decisions in order to manage order picking activities efficiently. This section discusses the practical implications of this research for warehouse managers.

Compared to the benchmark (i.e. strict order picking in combination with random storage assignment, FCFS batching, and aisle-by-aisle routing), all proposed combinations perform better. Over the 30 replications, the benchmark results in an average travel distance of 58,983.89 meter. The order picking process can be performed $76.9 \%$ more efficiently by dividing the warehouse into four order picking zones in combination with customer type zone assignment, within-aisle storage location assignment, seed batching, and optimal routing. This combination results in a mean route length of $13,608.14$ meter. As the simulation experiments have focused on operational order picking policy decisions only, the proposed combinations are rather easy to implement and result in large performance benefits.

We should note that maintaining order integrity can not be generalized to all warehouses as not all warehouses can divide their orders across customer types. However, even when SKUs are assigned to zones base on pick frequency, the average route length can be reduced with $72.6 \%$ compared to the benchmark. The mean route length for pick frequency zone assignment is minimized in combination with four zones, within-aisle storage location assignment, seed batching, and optimal routing, and results in an average travel distance of 16,147.10 meter.

In addition, the study of storage, batching, zone picking, and routing policies allows warehouse managers to determine the relations between order picking policies. The simulations provide insights into some excellent performing combinations (e.g. 2 zones (CT) and seed batching), as well as several inefficient policy combinations (e.g. FCFS batching and return routing). Furthermore, all main effects as well as all interaction effects have proven to be statistically significant. This implicates that warehouse managers should consider decisions on storage, batching, zoning, and routing simultaneously in order to minimize the distance traveled by order pickers and consequently reduce the order throughput time. Warehouse managers should be aware of the strong relations between order picking policies in order to optimize the overall warehouse performance.

\section{Conclusions}

The delivery of e-commerce markets forces warehouses to handle a growing number of orders in shorter time windows. Awareness of the influence of an individual warehouse operation on the overall warehouse performance is required to manage warehouse operations, resulting in enhanced customer service. 
In this paper, the relation between storage, batching, zone picking, and routing policies is studied for the first time. The results of the study clearly indicate that warehouses can achieve significant benefits by considering storage, batching, zone picking, and routing policies simultaneously. The combination of withinaisle storage, seed batching, zone picking (4 zones and customer type zone assignment), and the optimal route results in the lowest route length. As traveling is the dominant factor in order picking operations, the order picking process can be performed more efficiently. As a result, warehouse managers can either reduce the number of order pickers or reduce the customer order throughput time.

The simulation results of our study contribute to both practitioners and academic research. The evaluation of an extensive range of order picking policies helps warehouse managers to reduce the order throughput time. The simulated order picking policies can be easily implemented and immediately result in significant performance benefits. This paper is limited to the four main order picking policy decisions. However in future research, these order picking policies could be enlarged to other operational decisions, such as workforce planning decisions and alternative zone configurations. Furthermore, simulating other warehouse layouts may allow us to achieve higher practical and managerial relevance. Enlarging the simulation experiment will provide insight into more warehouse policy interactions, helping warehouse managers to further reduce the order throughput time, which can result in faster deliveries.

Acknowledgments. This work is supported by the Interuniversity Attraction Poles Programme initiated by the Belgian Science Policy Office (research project COMEX, Combinatorial Optimization: Metaheuristics \& Exact Methods).

\section{References}

1. Çelik, M., Süral, H.: Order picking under random and turnover-based storage policies in fishbone aisle warehouses. IIE Transactions 46(3), 283-300 (2014)

2. Chen, C.M., Gong, Y., de Koster, R.B., van Nunen, J.A.: A Flexible Evaluative Framework for Order Picking Systems. Production \& Operations Management 19(1), 70-82 (2010)

3. Chen, T.L., Cheng, C.Y., Chen, Y.Y., Chan, L.K.: An efficient hybrid algorithm for integrated order batching, sequencing and routing problem. International Journal of Production Economics 159, 158-167 (2015)

4. Cheng, C.Y., Chen, Y.Y., Chen, T.L., Jung-Woon Yoo, J.: Using a hybrid approach based on the particle swarm optimization and ant colony optimization to solve a joint order batching and picker routing problem. International Journal of Production Economics 170, Part C, 805-814 (2015)

5. Cohen, B.H., Welkowitz, J., Lea, R.B.: Introductory Statistics for the Behavioral Sciences (7th Edition). John Wiley \& Sons, Hoboken, NJ, USA (2011)

6. De Koster, R.B.M., Le-Duc, T., Roodbergen, K.J.: Design and control of warehouse order picking: A literature review. European Journal of Operational Research 182(2), 481-501 (2007)

7. Field, A.: Discovering Statistics using IBM SPSS Statistics. SAGE (2013) 
8. Geisser, S., Greenhouse, S.W.: An extension of box's results on the use of the $\mathrm{f}$ distribution in multivariate analysis. The Annals of Mathematical Statistics 29(3), 885-891 (1958)

9. Gu, J., Goetschalckx, M., McGinnis, L.F.: Research on warehouse operation: A comprehensive review. European Journal of Operational Research 177(1), 1-21 (2007)

10. Helsgaun, K.: An effective implementation of the LinKernighan traveling salesman heuristic. European Journal of Operational Research 126(1), 106-130 (2000)

11. Henn, S.: Algorithms for on-line order batching in an order picking warehouse. Computers \& Operations Research 39(11), 2549-2563 (2012)

12. Ho, Y.C., Tseng, Y.Y.: A study on order-batching methods of order-picking in a distribution centre with two cross-aisles. International Journal of Production Research 44(17), 3391-3417 (2006)

13. Ho, Y.C., Su, T.S., Shi, Z.B.: Order-batching methods for an order-picking warehouse with two cross aisles. Computers \& Industrial Engineering 55(2), 321-347 (2008)

14. Jane, C.C., Laih, Y.W.: A clustering algorithm for item assignment in a synchronized zone order picking system. European Journal of Operational Research 166(2), 489-496 (2005)

15. Petersen, C.G., Aase, G.: A comparison of picking, storage, and routing policies in manual order picking. International Journal of Production Economics 92(1), 11-19 (2004)

16. Petersen, C.G., Schmenner, R.W.: An Evaluation of Routing and Volume-based Storage Policies in an Order Picking Operation. Decision Sciences 30(2), 481-501 (1999)

17. Roodbergen, K.J.: Storage Assignment for Order Picking in Multiple-Block Warehouses. In: Manzini, R. (ed.) Warehousing in the Global Supply Chain, pp. 139-155. Springer London (2012)

18. Roodbergen, K.J., de Koster, R.B.M.: Routing methods for warehouses with multiple cross aisles. International Journal of Production Research 39(9), 1865-1883 (2001)

19. Theys, C., Bräysy, O., Dullaert, W., Raa, B.: Using a TSP heuristic for routing order pickers in warehouses. European Journal of Operational Research 200(3), $755-763$ (2010)

20. Won, J., Olafsson, S.: Joint order batching and order picking in warehouse operations. International Journal of Production Research 43(7), 1427-1442 (2005)

21. Yu, M., de Koster, R.B.M.: The impact of order batching and picking area zoning on order picking system performance. European Journal of Operational Research 198(2), 480-490 (2009) 